\title{
Carrier-carrier relaxation kinetics in quantum well semiconductor structures with nonparabolic energy bands
}

Dery, H.; Tromborg, Bjarne; Eisenstein, G.

Published in:

Physical Review B Condensed Matter

Link to article, DOI:

10.1103/PhysRevB.68.165323

Publication date:

2003

Document Version

Publisher's PDF, also known as Version of record

Link back to DTU Orbit

Citation (APA):

Dery, H., Tromborg, B., \& Eisenstein, G. (2003). Carrier-carrier relaxation kinetics in quantum well semiconductor structures with nonparabolic energy bands. Physical Review B Condensed Matter, 68(16), 165323. https://doi.org/10.1103/PhysRevB.68.165323

\section{General rights}

Copyright and moral rights for the publications made accessible in the public portal are retained by the authors and/or other copyright owners and it is a condition of accessing publications that users recognise and abide by the legal requirements associated with these rights.

- Users may download and print one copy of any publication from the public portal for the purpose of private study or research.

- You may not further distribute the material or use it for any profit-making activity or commercial gain

- You may freely distribute the URL identifying the publication in the public portal 


\title{
Carrier-carrier relaxation kinetics in quantum well semiconductor structures with nonparabolic energy bands
}

\author{
H. Dery, ${ }^{1, *}$ B. Tromborg, ${ }^{2}$ and G. Eisenstein ${ }^{1}$ \\ ${ }^{1}$ Department of Electrical Engineering, Technion-Israel Institute of Technology, Haifa 32000, Israel \\ ${ }^{2}$ Research Center COM, Technical University of Denmark, Building 345V, DK-2800 Lyngby, Denmark
}

(Received 27 January 2003; published 20 October 2003)

\begin{abstract}
We describe carrier-carrier scattering dynamics in an inverted quantum well structure including the nonparabolic nature of the valance band. A solution of the semiconductor Bloch equations yields strong evidence to a large change in the temporal evolution of the carrier distributions compared to the case of parabolic bands. The nonparabolic bands and the consequent change in the density of states reduce considerably the degree of gain saturation while decreasing the time constant governing the relaxation. This results in a measurable reduction of the role played by carrier-carrier scattering in determining the gain nonlinearity and hence the modulation and switching speed of lasers and optical amplifiers.
\end{abstract}

DOI: 10.1103/PhysRevB.68.165323

PACS number(s): 85.35.Be, 73.63.Hs, 42.55.Px

\section{INTRODUCTION}

The dynamics governing inverted semiconductors plays a major role in quantum structure based lasers and amplifiers. A common way to theoretically study gain and carrier dynamics is to calculate the evolution that follows a short pulse perturbation in a structure which is highly excited as in lasers or optical amplifiers. Many results can be obtained from relatively simple models but rigorous details require more complete formalisms, the two most common being the nonequilibrium Green's function theory ${ }^{1}$ and the density-matrix theory. ${ }^{2-4}$

On a subpicosecond time scale, the dominant dynamical process is carrier-carrier (CC) scattering. The present paper employs the density-matrix formalism to calculate the subpicosecond dynamics in a separate confinement heterostructure (SCH) strained quantum well structure. Our calculations extend the models available in the literature as we include the effect of nonparabolic bands. That is, we compare the relaxation processes under the conventional parabolic $E-k$ approximation to the case of nonparabolic bands. The nonparabolic nature of the bands turns out to have a major impact as it reduces the degree of gain saturation and shortens the time constant governing the recovery.

The inclusion of the nonparabolic bands clearly leads to an increased accuracy in the description of this widely researched topic. ${ }^{5-9}$ The moderation of the role played by spectral hole burning has also a major influence on the gain nonlinearity of quantum well $(\mathrm{QW})$ lasers and optical amplifiers and hence on their modulation and switching capabilities.

This paper is organized as follows. In the following section we briefly present the theoretical approach based on the density-matrix theory, along with mathematical aspects of the semiconductor Bloch equation (SBE). In Sec. III we present simulation results of the SBE. We address the scattering rate equations in the Appendix and elaborate on related mathematical issues concerning direct versus exchange scattering. We also present a brief discussion on the computational demands.

\section{THEORETICAL MODEL}

In the following analysis, we use the density-matrix approach along with the second quantization (SQ). The system Hamiltonian which includes noninteraction, radiation-matter interaction, and Coulomb interaction terms is calculated in the SQ representation using standard techniques.

$$
\begin{gathered}
H_{N I}=\sum_{\mathbf{k}}\left(\varepsilon_{e, k} a_{\mathbf{k}}^{\dagger} a_{\mathbf{k}}+\varepsilon_{h, k} b_{\mathbf{k}}^{\dagger} b_{\mathbf{k}}\right), \\
H_{r-m}=-\sum_{\mathbf{k}}\left[\mathbf{E}(t) \cdot \mu_{\mathbf{k}} a_{\mathbf{k}}^{\dagger} b_{-\mathbf{k}}^{\dagger}+\mathbf{E}^{*}(t) \cdot \mu_{\mathbf{k}}^{*} b_{-\mathbf{k}} a_{\mathbf{k}}\right], \\
H_{C}=\frac{1}{2} \sum_{\mathbf{k}, \mathbf{k}^{\prime}, \mathbf{q}} V_{q}\left(a_{\mathbf{k}+\mathbf{q}}^{\dagger} a_{\mathbf{k}^{\prime}-\mathbf{q}}^{\dagger} a_{\mathbf{k}^{\prime}} a_{\mathbf{k}}+b_{\mathbf{k}+\mathbf{q}}^{\dagger} b_{\mathbf{k}^{\prime}-\mathbf{q}^{\prime}}^{\dagger} b_{\mathbf{k}^{\prime}} b_{\mathbf{k}}\right. \\
\left.-2 a_{\mathbf{k}+\mathbf{q}}^{\dagger} b_{\mathbf{k}^{\prime}-\mathbf{q}}^{\dagger} b_{\mathbf{k}^{\prime}} a_{\mathbf{k}}\right),
\end{gathered}
$$

where $\varepsilon_{\lambda, k}$ denotes the single-particle energy in the state $(\lambda, \mathbf{k}), \mathbf{E}(t)$ is the electric field of the optical pulse, and $\mu_{\mathbf{k}}$ is the dipole matrix element. $V_{q}$ represents the bare Coulomb matrix element. The bare and static screened Coulomb matrix elements in heterostructures are derived and discussed in the appendix of Ref. 9.

\section{A. Semiconductor Bloch equations}

Three coupled differential equations comprise the SBE. Two equations describe the electron and hole distributions, and the third addresses the interband polarizations induced by the optical transitions.

$$
\begin{gathered}
\frac{d}{d t} n_{e, \mathbf{p}}=\left.\frac{d}{d t} n_{e, \mathbf{p}}\right|_{c o l}+i\left[\Omega_{\mathbf{p}} p_{\mathbf{p}}^{*}-\Omega_{\mathbf{p}}^{*} p_{\mathbf{p}}\right], \\
\frac{d}{d t} n_{h, \mathbf{p}}=\left.\frac{d}{d t} n_{h, \mathbf{p}}\right|_{c o l}+i\left[\Omega_{\mathbf{p}} p_{\mathbf{p}}^{*}-\Omega_{\mathbf{p}}^{*} p_{\mathbf{p}}\right], \\
\frac{d}{d t} p_{\mathbf{p}}=\left.\frac{d}{d t} p_{\mathbf{p}}\right|_{c o l}-i \omega_{\mathbf{p}} p_{\mathbf{p}}+i \Omega_{\mathbf{p}}\left[1-n_{h, \mathbf{p}}-n_{e, \mathbf{p}}\right],
\end{gathered}
$$



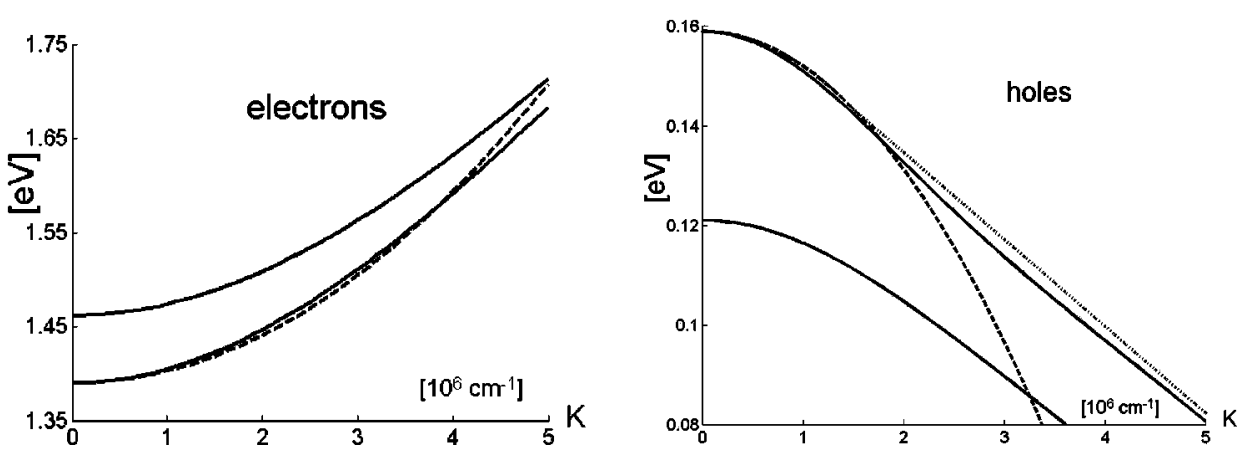

FIG. 1. $E-k$ dispersions of the first and second subbands as computed from the 8-band Kane model using the $k \cdot p$ theory are given by solid lines. Left: electrons. Right: holes. The dashed lines give the parabolic approximation of the first subband, where we used the mass of the $\Gamma$ point $\left(m_{e}=0.03 m_{0}, m_{h}=0.055 m_{0}\right)$. The dotted line is the nonparabolic first subband functional extrapolation of the holes.

$$
\begin{gathered}
\hbar \omega_{\mathbf{p}}=\left(\varepsilon_{e, \mathbf{p}}+\varepsilon_{h, \mathbf{p}}\right)-\sum_{\mathbf{q}} V_{q}\left(n_{e, \mathbf{p}+\mathbf{q}}+n_{h, \mathbf{p}+\mathbf{q}}\right), \\
\Omega_{\mathbf{p}}=\frac{\mathbf{E}(t) \cdot \mu_{\mathbf{p}}}{\hbar}+\frac{1}{\hbar} \sum_{\mathbf{q}} V_{q} p_{\mathbf{p}+\mathbf{q}},
\end{gathered}
$$

where $\omega_{\mathbf{p}}$, and $\Omega_{\mathbf{p}}$ denote, respectively, the renormalized transition and Rabi frequencies. Equations (4)-(8) are the SBE for the interacting particles model. The collisional contribution is

$$
\begin{aligned}
\left.\frac{d}{d t} n_{\alpha, \mathbf{p}}\right|_{c o l}= & \left(\Gamma_{\alpha, \mathbf{p}}^{\text {in }}+\Delta_{\alpha, \mathbf{p}}^{\text {in,(2) }}\right)\left(1-n_{\alpha, \mathbf{p}}\right)-\left(\Gamma_{\alpha, \mathbf{p}}^{\text {out }}+\Delta_{\alpha, \mathbf{p}}^{\text {out },(2)}\right) n_{\alpha, \mathbf{p}} \\
& +\left(\Delta_{\alpha, \mathbf{p}}^{(1)} p_{\mathbf{p}}+\text { c.c. }\right) \\
\left.\frac{d}{d t} p_{\mathbf{p}}\right|_{c o l}= & \sum_{\alpha}\left[-\left(\Gamma_{\alpha, \mathbf{p}}^{\text {in }}+\Gamma_{\alpha, \mathbf{p}}^{\text {out }}\right) p_{\mathbf{p}}+\Lambda_{\alpha, \mathbf{p}}^{\text {in,(1) }}\left(1-n_{\alpha, \mathbf{p}}\right)\right. \\
& \left.+\Lambda_{\alpha, \mathbf{p}}^{\text {out,(1) }} n_{\alpha, \mathbf{p}}+\Lambda_{\alpha, \mathbf{p}}^{(2)} p_{\mathbf{p}}+\Lambda_{\alpha, \mathbf{p}}^{(3)}\right]
\end{aligned}
$$

where the superscripts in bracket of the rates $\Delta, \Lambda$ refer to the polarization power. For relatively weak optical pulses, the terms involving $\Delta^{(1)}, \Delta^{(2)}, \Lambda^{(2)}, \Lambda^{(3)}$ are negligible (these involve the square and third power polarization). In all rate terms, exchange contributions are considered. The explicit form of these rates is given in the appendix.

\section{B. Numerical aspects}

The SBE given by Eqs. (4)-(10) were solved for a typical SCH QW laser amplifier with the following parameters. The total width of the heterostructure was $985 \AA$ including a single $\mathrm{In}_{.25} \mathrm{Ga}_{.75} \mathrm{As} 85 \AA$ wide well with $100 \AA$ GaAs barriers on each side. The rest of the structure consists of $\mathrm{Al}_{.18} \mathrm{Ga}_{.82} \mathrm{As}$ on either sides. The temperature was fixed at $300 \mathrm{~K}$. The well is subjected to $1.8 \%$ compressive strain due to the $7.2 \%$ lattice mismatch between GaAs and InAs. The $E-k$ diagrams and the confined wave functions were computed following Ref. 10. The particle masses at the $\Gamma$ point $(\mathbf{k}=\mathbf{0})$ and Coulomb matrix elements were extracted from these data:

$$
m_{e}=0.03 m_{0}, \quad m_{h}=0.055 m_{0},
$$

$$
E_{g}=E_{e}-E_{h}=1.2312 \mathrm{eV} .
$$

We simulate a medium that is excited by a 75-fs Gaussianshaped optical pulse (the un-normalized Rabi frequency at the peak of the pulse $\mathbf{E}_{0} \cdot \mu_{\mathbf{k}} / \hbar$ was $\approx 7.5 \times 10^{12} \mathrm{~Hz}$ ). The amplifier is assumed to be short, namely, we do not consider pulse propagation effects or any distributed nonlinearities.

At "infinite time" before the arrival of the pulse peak, the polarization inside the medium is assumed to be zero throughout and the initial subband distributions are quasithermal Fermi-Dirac distributions within the bands. The total carrier density was taken as $N_{2 d}=10^{12} \mathrm{~cm}^{-2}$.

\section{RESULTS AND DISCUSSION}

Figure 1 shows the $E$ - $k$ dispersions for electrons (left) and holes (right). The solid lines are the result of an 8-band Kane model using the $k \cdot p$ theory. We have plotted the first two subbands at each band where for the holes strain induces heavy-hole-light-hole splitting. The dashed lines denote the parabolic approximation of the first subbands, where we used the mass of the $\Gamma$ point $\left(m_{e}=0.03 m_{0}, m_{h}=0.055 m_{0}\right)$. The dotted line shows the first subband nonparabolic functional extrapolation of the holes. The difference between the parabolic and nonparabolic curves is very evident. At elevated densities, typical to optical amplifiers and lasers, the deviation is nonnegligible in the energy regions where the probability is not very small. The nonparabolic functional form of the $E-k$ curvature was approximated by an extrapolation. In the low-wave-vector region we used a parabolic form whereas for high wave-vectors, we assumed a linear function. The two regions were joined using a smoothing procedure. The resultant functional form is given by the dotted line of Fig. 1.

Figure 2 shows a calculated time evolution of the carrier distributions according to Eqs. (4)-(10). The two left graphs denote electron (upper left graph) and hole (lower left graph) distributions calculated using parabolic dispersion curves. The right graphs correspond to calculations using the nonparabolic dispersion. Each graph comprises five curves describing the distributions at times $-\infty \mathrm{fs}$, upper solid; $-40 \mathrm{fs}$, upper dashed; 80 fs, lower solid; 250 fs, lower dashed; and the middle solid curve denotes $1.2 \mathrm{ps}$ (700 fs) in the parabolic (nonparabolic) case. The reference time $t=0 \mathrm{fs}$ is 

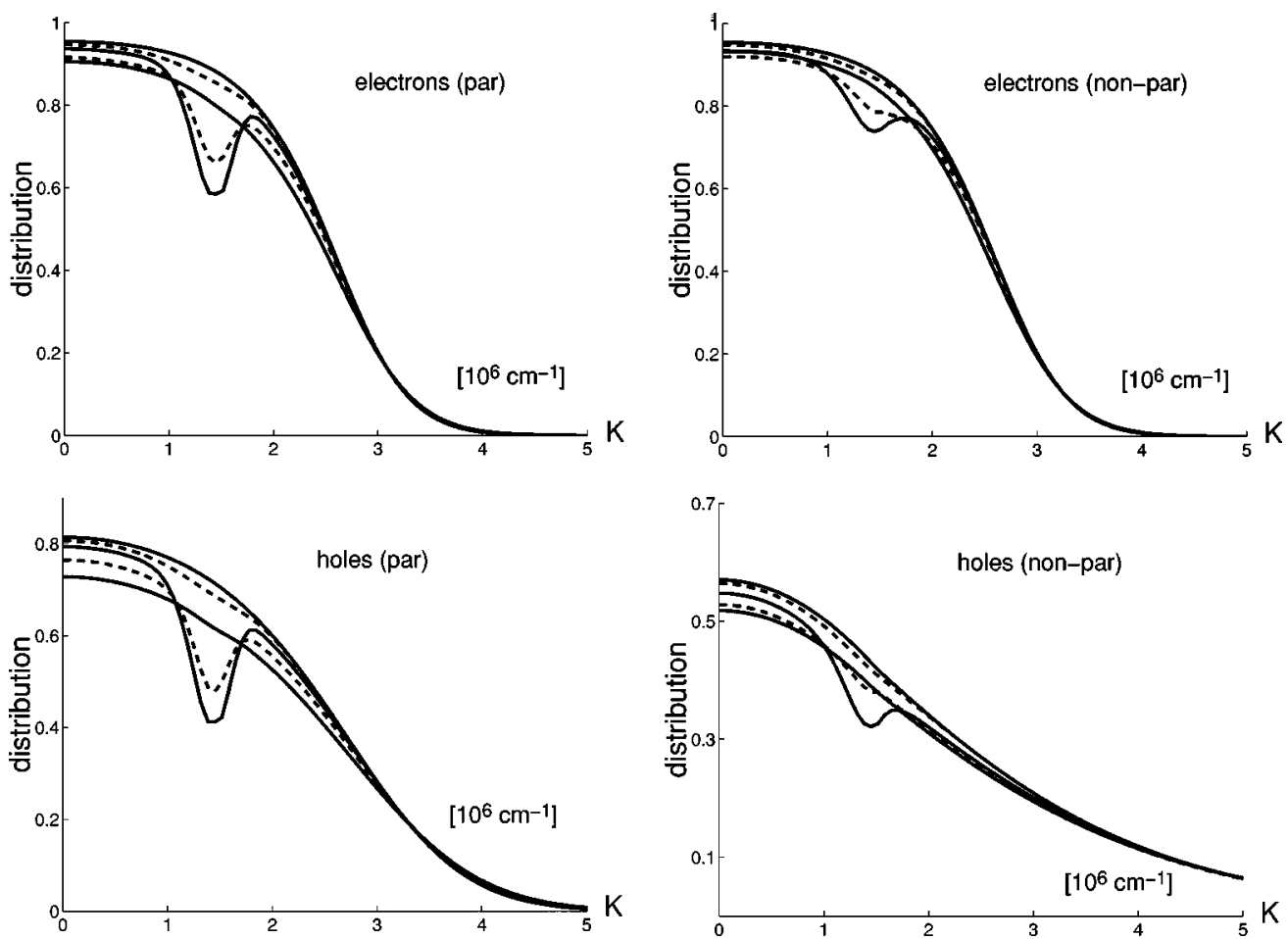

FIG. 2. Evolution of electron and hole distributions. Upper: electrons. Lower: holes. Left: parabolic. Right: nonparabolic. In each figure the upper solid line denotes the initial distribution $-\infty$ fs before the arrival of the pulse peak. Upper dashed line denotes the distribution at $-40 \mathrm{fs}$, lower solid $+80 \mathrm{fs}$ at which the plasma is the most perturbed, lower dashed $+250 \mathrm{fs}$. The middle solid line denotes $+1.2 \mathrm{ps}$ $(+700 \mathrm{fs})$ in the parabolic (nonparabolic) case.

taken at the peak of the arriving pulse.

Figure 2 highlights two clear observations. First, the system deviation from equilibrium due to the perturbing pulse is lower in the nonparabolic case, and second the relaxation time is shortened appreciatively. The diminished deviation from equilibrium also reduces the plasma heating effect. The influence on the carrier dynamics of the nonparabolic bands amounts to a reduction of gain saturation effects and hence an improvement of the modulation and switching capabilities. In order to quantify the reduction in the nonlinear gain, we assumed that $700 \mathrm{fs}$ after the perturbing pulse, the carriers reach Fermi-Dirac distributions. We can then extract the chemical potential and the electron temperature from the first and third wave-vector moments (corresponding for the total density and the energy).

The simulation compares only the electron distributions in the two cases because, unlike the holes, their initial distributions are identical. The temperature of the electrons was 325 $\mathrm{K}$ in the nonparabolic case and $350 \mathrm{~K}$ in the parabolic case. The chemical-potential variation was also doubled in the parabolic case. This also may indicate that carrier heating effect is also reduced in the nonparabolic case.

From a physical point of view, the nonparabolic dispersion changes the system density of states and hence the rate of carrier transfer between arbitrary initial and final states $\left(\gamma_{i, f}=\gamma_{i, j}^{0}\left[\left(1-n_{f}\right) n_{i}-\left(1-n_{i}\right) n_{f}\right]\right)$ is affected by the different distributions and also by the different $\gamma_{i, j}^{0}$. In our case where the $\mathrm{CC}$ scattering determines the relaxation rate, the increase of holes population in higher-energy states increases the probability for carriers to scatter with these holes.
Mathematically, each of the rate terms in Eqs. (9) and (10) involves energy conservation denoted by Dirac $\delta$ function (see the Appendix). The absolute value of the derivative at the zeros of the $\delta$ argument appears in the denominators of each rate term during the integration process. The milder slope (which is associated to the energy derivative) of the nonparabolic dispersion increases the relative weight of the rate in these cases resulting in a faster relaxation. The difference in slopes is seen in the dashed and dotted lines describing the holes in Fig. 1.

The momentum Region in Fig. 2 where the plasma is most perturbed was also examined by testing the time evolution of the electrons and the absolute value of the polarization. The values of these entities are directly related to ultrafast pumpprobe and four wave mixing experiments in optical amplifiers and lasers which explore the relation between carrier dynamics and optical gain in the subsecond pico time scale. The left graph of Fig. 3 shows the electron distribution versus time in this momentum region. The right graph shows the corresponding time evolution of the absolute value of the polarization. The solid lines represent the nonparabolic bands while the dashed curves are for the conventional parabolic case. $t=0$ fs refers in this figure to $-150 \mathrm{fs}$ before the peak arrival. As the spectral hole is formed, carrier-carrier scattering acts as a restoring force that tries to equilibrate the system. This effect is significantly weaker in the parabolic case and the pulse saturates the gain to a degree where the system reaches the transparency point. Figure 2 reveals that in the most perturbed case, $n_{h, \mathbf{k}_{\mathbf{1}}}$ 

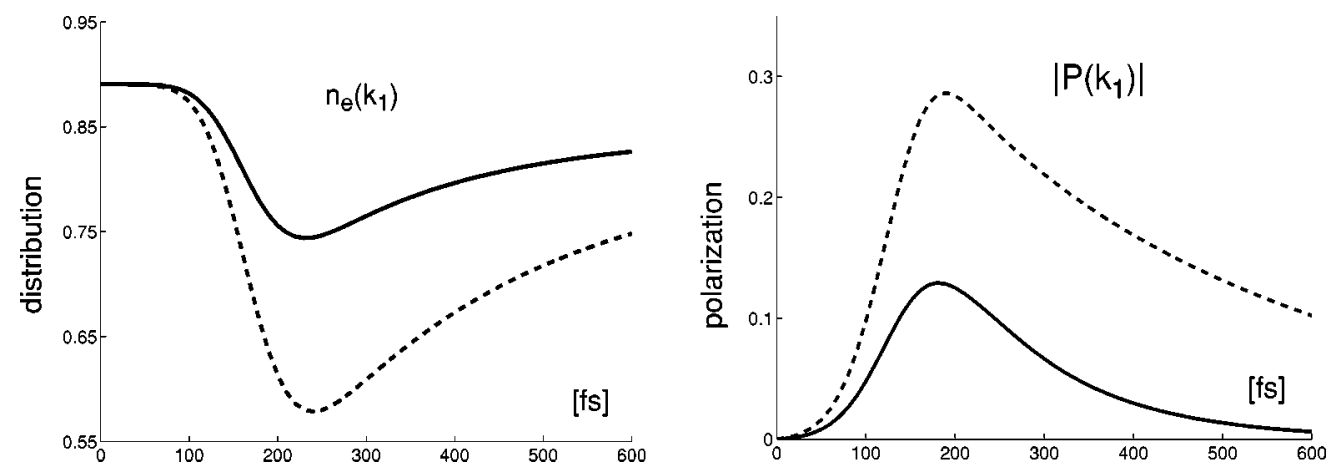

FIG. 3. In both graphs we have considered the momentum region where the plasma is most perturbed. Left: the evolution of the electrons distribution. Right: the absolute value of the polarization vs time. The nonparabolic (parabolic) cases are denoted by solid (dashed) lines.

$+n_{h, \mathbf{k}_{\mathbf{1}}} \rightarrow 1$; whereas in the nonparabolic case, it is larger than 1 .

At later times, when the plasma experiences only the trailing tail of the pulse, the curves of Fig. 3 can be approximated by single exponential decay time $[a+b \exp (-t / \tau)]$. For both the electron evolution and the absolute polarization, the time constant governing the decay of the nonparabolic case was $\tau=190 \mathrm{fs}$ and for the parabolic bands it was $\tau=260 \mathrm{fs}$. The difference in decay rates is larger near the peak of the arriving pulse where a single time constant can, of course, not be extracted.

\section{ACKNOWLEDGMENTS}

H.D. acknowledges financial support from the Levi Eshkol. This research was partially supported by the Israeli Science Foundation.

\section{APPENDIX A: COLLISION RATES}

In the following scattering rate terms, if $\alpha$ denotes the conduction band then $\beta$ denotes the valence band and vice versa. One can get the out-scattering rate terms $X_{\lambda, \mathbf{p}}^{\text {out }}$ by replacing $n_{\lambda, \mathbf{p}} \leftrightarrow 1-n_{\lambda, \mathbf{p}}$ in Eqs. (A5), (A6), and (A8). In order to explicitly write the form of the rate terms in Eqs. (9) and (10), we introduce the following notations:

$$
\begin{gathered}
\widetilde{V}_{\mathbf{k}, \mathbf{p}, \mathbf{q}}^{1}=V_{s, q}-V_{s,|\mathbf{k}-\mathbf{p}-\mathbf{q}|} \\
\widetilde{V}_{\mathbf{k}, \mathbf{p}, \mathbf{q}}^{2}=V_{s, q}^{*} \cdot \widetilde{V}_{\mathbf{k}, \mathbf{p}, \mathbf{q}}^{1}, \\
\delta^{1}(\alpha, \mathbf{p}, \mathbf{q}, \mathbf{k})=\delta\left(\varepsilon_{\alpha, \mathbf{p}+\mathbf{q}}+\varepsilon_{\alpha, \mathbf{k}-\mathbf{q}}-\varepsilon_{\alpha, \mathbf{k}}-\varepsilon_{\alpha, \mathbf{p}}\right), \\
\delta^{2}(\alpha, \mathbf{p}, \mathbf{q}, \mathbf{k})=\delta\left(\varepsilon_{\alpha, \mathbf{p}+\mathbf{q}}-\varepsilon_{\beta, \mathbf{k}-\mathbf{q}}+\varepsilon_{\beta, \mathbf{k}}-\varepsilon_{\alpha, \mathbf{p}}\right), \\
\Gamma_{\alpha, \mathbf{p}}^{i n}=\frac{2 \pi}{\hbar} \sum_{\mathbf{k}, \mathbf{q}} n_{\alpha, \mathbf{p}+\mathbf{q}}\left[\delta^{1}(\alpha, \mathbf{p}, \mathbf{q}, \mathbf{k}) \cdot \widetilde{V}_{\mathbf{k}, \mathbf{p}, \mathbf{q}}^{2} \cdot(1\right. \\
\left.-n_{\alpha, \mathbf{k}}\right) \cdot n_{\alpha, \mathbf{k}-\mathbf{q}}+\delta^{2}(\alpha, \mathbf{p}, \mathbf{q}, \mathbf{k}) \cdot\left|V_{s, q}\right|^{2} \cdot(1 \\
\left.\left.-n_{\beta, \mathbf{k}-\mathbf{q}}\right) \cdot n_{\beta, \mathbf{k}}\right]
\end{gathered}
$$

$$
\begin{aligned}
& \Delta_{\alpha, \mathbf{p}}^{i n,(2)}=\frac{-\pi}{\hbar} \sum_{\mathbf{k}, \mathbf{q}}\left(n _ { \alpha , \mathbf { p } + \mathbf { q } } \left[\widetilde{V}_{\mathbf{k}, \mathbf{p}, \mathbf{q}}^{2} \cdot p_{\mathbf{k}-\mathbf{q}}^{*} \cdot p_{\mathbf{k}}\right.\right. \\
& + \text { c.c. }] \cdot\left[\delta^{1}(\alpha, \mathbf{p}, \mathbf{q}, \mathbf{k})+\delta^{2}(\alpha, \mathbf{p}, \mathbf{q}, \mathbf{k})\right] \\
& +\delta^{2}(\alpha, \mathbf{p}, \mathbf{q}, \mathbf{k}) \cdot n_{\beta, \mathbf{k}} \cdot\left[V_{s, q}^{*} \cdot V_{s,|\mathbf{k}-\mathbf{p}-\mathbf{q}|} \cdot p_{\mathbf{k}-\mathbf{q}}^{*} \cdot p_{\mathbf{p}+\mathbf{q}}\right. \\
& \text { +c.c.]), } \\
& \Delta_{\alpha, \mathbf{p}}^{(1)}=\frac{\pi}{\hbar} \sum_{\mathbf{k}, \mathbf{q}}\left\{\widetilde { V } _ { \mathbf { k } , \mathbf { p } , \mathbf { q } } ^ { 2 } \cdot p _ { \mathbf { p } + \mathbf { q } } ^ { * } \cdot \left[\delta^{1}(\alpha, \mathbf{p}, \mathbf{q}, \mathbf{k}) \cdot\left(n_{\alpha, \mathbf{k}}-n_{\alpha, \mathbf{k}-\mathbf{q}}\right)\right.\right. \\
& \left.+\delta^{2}(\alpha, \mathbf{p}, \mathbf{q}, \mathbf{k}) \cdot\left(n_{\beta, \mathbf{k}-\mathbf{q}}-n_{\beta, \mathbf{k}}\right)\right] \\
& +\delta^{2}(\alpha, \mathbf{p}, \mathbf{q}, \mathbf{k}) \cdot\left(n_{\beta, \mathbf{k}-\mathbf{q}}\right. \\
& \left.\left.-n_{\alpha, \mathbf{p}+\mathbf{q}}\right) \cdot V_{s, q}^{*} \cdot V_{s,|\mathbf{k}-\mathbf{p}-\mathbf{q}|} \cdot p_{\mathbf{k}}^{*}\right\}, \\
& \Lambda_{\alpha, \mathbf{p}}^{i n,(1)}=\frac{\pi}{\hbar} \sum_{\mathbf{k}, \mathbf{q}}\left(\widetilde { V } _ { \mathbf { k } , \mathbf { p } , \mathbf { q } } ^ { 2 } \cdot p _ { \mathbf { p } + \mathbf { q } } \cdot \left[\delta^{2}(\beta, \mathbf{p}, \mathbf{q}, \mathbf{k}) \cdot(1\right.\right. \\
& \left.\left.-n_{\alpha, \mathbf{k}}\right) \cdot n_{\alpha, \mathbf{k}-\mathbf{q}}+\delta^{1}(\beta, \mathbf{p}, \mathbf{q}, \mathbf{k}) \cdot\left(1-n_{\beta, \mathbf{k}-\mathbf{q}}\right) \cdot n_{\beta, \mathbf{k}}\right] \\
& +\delta^{2}(\beta, \mathbf{p}, \mathbf{q}, \mathbf{k}) \cdot n_{\alpha, \mathbf{k}-\mathbf{q}} \cdot(1 \\
& \left.\left.-n_{\beta, \mathbf{p}+\mathbf{q}}\right) \cdot V_{s, q}^{*} \cdot V_{s,|\mathbf{k}-\mathbf{p}-\mathbf{q}|} \cdot p_{\mathbf{k}}\right), \\
& \Lambda_{\alpha, \mathbf{p}}^{(2)}=\frac{\pi}{\hbar} \sum_{\mathbf{k}, \mathbf{q}}\left(\tilde { V } _ { \mathbf { k } , \mathbf { p } , \mathbf { q } } ^ { 2 } \cdot \left[\delta^{2}(\alpha, \mathbf{p}, \mathbf{q}, \mathbf{k}) \cdot p_{\mathbf{k}-\mathbf{q}}^{*} p_{\mathbf{k}}\right.\right. \\
& \left.+\delta^{1}(\alpha, \mathbf{p}, \mathbf{q}, \mathbf{k}) \cdot p_{\mathbf{k}-\mathbf{q}} p_{\mathbf{k}}^{*}\right] \\
& \left.+\delta^{2}(\beta, \mathbf{p}, \mathbf{q}, \mathbf{k}) \cdot V_{s, q}^{*} \cdot V_{|\mathbf{k}-\mathbf{p}-\mathbf{q}|} \cdot p_{\mathbf{k}-\mathbf{q}}^{*} p_{\mathbf{p}+\mathbf{q}}\right), \\
& \Lambda_{\alpha, \mathbf{p}}^{(3)}=\frac{-\pi}{\hbar} \sum_{\mathbf{k}, \mathbf{q}} p_{\mathbf{p}+\mathbf{q}}\left(\delta^{1}(\alpha, \mathbf{p}, \mathbf{q}, \mathbf{k}) \cdot \widetilde{V}_{\mathbf{k}, \mathbf{p}, \mathbf{q}}^{2} \cdot p_{\mathbf{k}-\mathbf{q}} p_{\mathbf{k}}^{*}\right. \\
& \left.+\delta^{2}(\beta, \mathbf{p}, \mathbf{q}, \mathbf{k}) \cdot\left|V_{s, q}^{*}\right|^{2} \cdot p_{\mathbf{k}-\mathbf{q}}^{*} p_{\mathbf{k}}\right) .
\end{aligned}
$$

Each of these collision integral expressions $(\Gamma, \Delta, \Lambda)$ denotes a rate which is computed by three integrations. For each $p$ the integration is held over $q$, over the angle between $\mathbf{p}$ and $\mathbf{q}(\varphi)$ and over $k$. In the Markovian approximation, the angle between $\mathbf{k}$ and $\mathbf{q}(\phi)$ is determined by the energy Dirac $\delta$ function. There are however subtle cases where the integrand 
diverges (longitudinal scattering: $\mathbf{k} \cdot \mathbf{q}= \pm k q$ ). These divergences can be removed by variable manipulations, which change the limits of the $k$ integration. There are two possible $\phi$ angles for each $k\left(\phi_{k}\right.$ and $\left.2 \pi-\phi_{k}\right)$. The direct terms are the same for both angles because their scalar product magnitudes are equal. These terms contain products of the form $\left|V_{s, q}\right|^{2}$. On the other hand, in the exchange terms that contain products of the form $V_{s, q}^{*} \cdot V_{s,|\mathbf{k}-\mathbf{p}-\mathbf{q}|}$, the magnitude of the vector $|\mathbf{k}-\mathbf{p}-\mathbf{q}|$ is affected differently by $\phi_{k}$ and $2 \pi$ $-\phi_{k}$ due to the angle between $\mathbf{k}$ and $\mathbf{p}(\varphi+\phi$ and $\varphi-\phi)$. The direct terms contain a factor of 2 whereas the exchange terms split into two cases. The $\varphi$ integration from 0 to $\pi$ and from $\pi$ to $2 \pi$ yields the same results for the direct and exchange cases, and hence one can integrate only over the first region and have an additional overall factor of 2 .

In addition to a detailed physical formalism, the results we have presented involve considerable computational complexities. All computer programs used to solve the SBE were written in $\mathrm{C}^{++}$, and can be described as CPU bounded. A memory bound program would have consumed memory over and above the RAM limit what would have caused a bottleneck due to frequent disk access. The calculations were performed using the Origin 2001 at the HPCU center in TelAviv. The simulations used 32 dedicated parallel SGI CPU's each operating at $500 \mathrm{MHz}$ and having a memory size beyond the program needs.
*Electronic address: dery @ techunix.technion.ac.il

${ }^{1}$ See, for example, H. Haug, in Optical Nonlinearities and Instabilities in Semiconductors, edited by H. Haug (Academic, San Diego, 1988), p. 53.

${ }^{2} \mathrm{~T}$. Kuhn, in Theory of Transport Properties of Semiconductor NanoStructures, edited by E. Schöll (Chapman \& Hall, London, 1998), pp. 173-214.

${ }^{3}$ See, for example, M. Lindberg and S.W. Koch, Phys. Rev. B 38, 3342 (1988).

${ }^{4}$ H.W. Wyld and B.D. Fried, Ann. Phys. (N.Y.) 23, 374 (1963).
${ }^{5}$ R. Binder, D. Scott, A.E. Paul, M. Lindberg, K. Henneberger, and S.W. Koch, Phys. Rev. B 45, 1107 (1992).

${ }^{6}$ See, for example, S. Haas, F. Rossi and T. Kuhn, Phys. Rev. B 53, 12855 (1996); S. Hughes, Phys. Rev. A 58, 2567 (1998).

${ }^{7}$ S.M. Goodnick and P. Lugli, Phys. Rev. B 37, 2578 (1988).

${ }^{8}$ F. Prengel and E. Schöll, Phys. Rev. B 59, 5806 (1999).

${ }^{9}$ H. Dery, B. Tromborg, and G. Eisenstein Phys. Rev. B 67, 245308 (2003).

${ }^{10}$ D. Gershoni, C.H. Henry, and G.A. Baraff, IEEE J. Quantum Electron. QE-29, 2433 (1993). 